\title{
Improvement of calcium balance by Fructus Ligustri Lucidi extract in mature female rats was associated with the induction of serum parathyroid hormone levels
}

\author{
Xiao-Li Dong ${ }^{1,2}$, Ming Zhao ${ }^{3}$, Kwun-Kit Wong ${ }^{1}$, Chun-Tao Che ${ }^{3,4}$ and Man-Sau Wong ${ }^{1,5 *}$ \\ ${ }^{1}$ Central Laboratory of the Institute of Molecular Technology for Drug Discovery and Synthesis, Department of Applied \\ Biology and Chemical Technology, The Hong Kong Polytechnic University, Hung Hom, Kowloon, Hong Kong, People's \\ Republic of China \\ ${ }^{2}$ Department of Medical Psychology and Psychiatry, Medical College of Qingdao University, Qingdao 266071, People's \\ Republic of China \\ ${ }^{3}$ School of Chinese Medicine, The Chinese University of Hong Kong, Hong Kong, People's Republic of China \\ ${ }^{4}$ Department of Medicinal Chemistry and Pharmacognosy, College of Pharmacy, University of Illinois at Chicago, Chicago, \\ IL 60612, USA \\ ${ }^{5}$ Shenzhen Research Institute of The Hong Kong Polytechnic University, State Key Laboratory of Chinese Medicine and \\ Molecular Pharmacology (Incubation), Shenzhen 518057, People's Republic of China
}

(Submitted 10 March 2011 - Final revision received 22 August 2011 - Accepted 22 August 2011 - First published online 12 October 2011)

\section{Abstract}

Fructus Ligustri Lucidi (FLL) is a commonly prescribed herb in many kidney-tonifying Traditional Chinese Medicinal formulae for the treatment of osteoporosis. The present study aimed to identify the active fractions in FLL and to characterise its effects on Ca balance, calciotropic hormone levels as well as bone properties in mature female rats fed diets containing different levels of Ca. In the present study, 4-month-old Sprague-Dawley female rats were treated with either FLL ethanol extract (EE), ethyl acetate-soluble fraction of EE (EAF), water-soluble fraction of EE (WF) or their vehicle for 12 weeks on a medium-Ca diet (MCD, $0.6 \% \mathrm{Ca}, 0.65 \% \mathrm{P})$. Then, the Sprague-Dawley female rats treated with WF or its vehicle for 12 weeks were fed diets containing different levels of dietary Ca (low-Ca diet (LCD), $0.1 \% \mathrm{Ca}, 0.65 \% \mathrm{P}$; MCD; high-Ca diet (HCD), $1.2 \% \mathrm{Ca}, 0.65 \% \mathrm{P}$ ). The results demonstrated that WF from EE but not EAF exerted a prominent effect on Ca balance by inhibiting urinary and faecal Ca excretion. WF significantly increased Ca balance in rats fed MCD or HCD with an associated increase in serum parathyroid hormone (PTH) levels. WF did not alter bone mineral density or bone mineral content of the tibia in all the rats fed with different levels of dietary $\mathrm{Ca}$. In conclusion, WF was responsible for the positive actions of FLL on $\mathrm{Ca}$ absorption and balance. The regulation of Ca balance by WF might involve its action in stimulating PTH production in the mature female rats.

Key words: Fructus Ligustri Lucidi: Calcium balance: Mature rats: Dietary calcium: Parathyroid hormone

Fructus Ligustri Lucidi (FLL), the fruit of Ligustrum lucidum Ait, is a commonly prescribed herb to nourish the endocrine and renal systems and to strengthen the bones in Traditional Chinese Medicine ${ }^{(1)}$. In the clinical practice of Traditional Chinese Medicine, FLL is a component of many kidneytonifying Chinese herbal formulae for the treatment of osteoporosis $^{(2)}$. Our previous study demonstrated that the ethanol extract (EE) of FLL could improve bone properties in the aged female rats, mainly by elevating Ca absorption and balance ${ }^{(2,3)}$. FLL was proven to have improved efficiency in utilising Ca from diet, mainly via its actions on increasing serum 1,25-dihydroxyvitamin $\mathrm{D}_{3}\left(1,25(\mathrm{OH})_{2} \mathrm{D}_{3}\right)$ level and vitamin $\mathrm{D}$-dependent $\mathrm{Ca}$ binding proteins $(\mathrm{CaBP})^{(2)}$. Our results demonstrated that FLL enhanced the transcellular $\mathrm{Ca}$ transport in aged female rats fed with sufficient dietary $\mathrm{Ca}^{(2)}$. Our recent study reported that FLL increased the circulating

Abbreviations: $1,25(\mathrm{OH})_{2} \mathrm{D}_{3}, 1,25$-dihydroxyvitamin $\mathrm{D}_{3} ; \mu \mathrm{CT}$, micro-computed tomography; BS, bone surface; BV, bone volume; C, control; CaBP, Ca binding proteins; Cr, creatinine; EAF, ethyl acetate-soluble fraction of ethanol extract; EE, ethanol extract; FLL, Fructus Ligustri Lucidi; HCD, high-Ca diet; LCD, low-Ca diet; MCD, medium-Ca diet; NDS, non-digestible saccharides; PTH, parathyroid hormone; TV, tissue volume; WF, water-soluble fraction of ethanol extract.

*Corresponding author: Dr M.-S. Wong, fax +852 23649932, email bcmswong@polyu.edu.hk 
levels of $1,25(\mathrm{OH})_{2} \mathrm{D}_{3}$ in aged female rats by directly stimulating the activity of its biosynthetic enzyme, 25-hydroxyvitamin D 1-hydroxylase ${ }^{(4)}$. Collectively, our research work on FLL indicated that direct actions of FLL EE on the vitamin D system might act as the initial targets for its protective effects on bone in the aged female rats ${ }^{(2,4)}$.

Ca balance is determined by the relationships between $\mathrm{Ca}$ intake, absorption and excretion. Ca homeostasis in vivo is regulated by calciotropic hormones, including parathyroid hormone (PTH) and $1,25(\mathrm{OH})_{2} \mathrm{D}_{3}$. PTH regulates Ca homeostasis through three aspects, namely, stimulation of Ca release from bone, stimulation of renal $\mathrm{Ca}$ reabsorption to decrease urinary $\mathrm{Ca}$ loss and stimulation of renal $1,25(\mathrm{OH})_{2} \mathrm{D}_{3}$ production $^{(5)}$. Ca (re)absorption through the intestine and kidney occurs by way of two main mechanisms, which are normally called paracellular and transcellular $\mathrm{Ca}$ transport. The paracellular transport is a passive non-saturable route ${ }^{(5)}$ while the transcellular transport is an active route that includes the action of PTH and vitamin D systems ${ }^{(6)}$. Intestinal Ca absorption and renal $\mathrm{Ca}$ reabsorption are primarily regulated by the genomic actions of $1,25(\mathrm{OH})_{2} \mathrm{D}_{3}$ on the transcellular pathway ${ }^{(6)}$ or through its non-genomic pathway on paracellular Ca transport ${ }^{(7,8)}$. PTH seems to act indirectly on intestinal Ca absorption by stimulating $1,25(\mathrm{OH})_{2} \mathrm{D}_{3}$ production $^{(5)}$, and the direct effect of PTH on the global process of intestinal $\mathrm{Ca}$ absorption has not yet been reported. PTH and $1,25(\mathrm{OH})_{2} \mathrm{D}_{3}$ interact to organise in a multilevel negative feedback loop for the purpose of maintaining Ca homeostasis.

Despite the fact that FLL could clearly modulate Ca and vitamin D metabolism in female rats, the chemical constituents in FLL that account for its positive effect on bone and mineral metabolism remain unknown. Previous studies ${ }^{(9-11)}$ reported that oleanolic acid and ursolic acid are the active compounds that might account for the hepatoprotective and osteoprotective effects of FLL. In addition, FLL contains quantitative constituents of polysaccharides. The polysaccharides in FLL, which are usually extracted by water ${ }^{(12)}$, are proven to exert anti-oxidation, anti-ageing ${ }^{(13)}$ and immune stimulation effects $^{(14)}$ in animal experiments. It is unclear if these previously reported compounds and constituents are responsible for the positive effects of FLL on Ca balance.

The present study aimed to determine the active fractions in FLL that are responsible for its positive effects on Ca balance and to characterise the effects of the FLL active fractions on Ca balance, calciotropic hormone levels as well as bone properties in mature female rats in response to treatment with diets containing different levels of $\mathrm{Ca}$. It is hoped that the present study will further increase our understanding of the molecular actions of the active fractions in FLL that might be useful for increasing Ca bioavailability.

\section{Methods}

\section{Preparation and fractionation of Fructus Ligustri Lucidi}

Fructus Ligustri Lucidi (FLL) was obtained from Jiangsu province of China. A voucher specimen was deposited in The Hong Kong Polytechnic University. The crude plant ( $40 \mathrm{~kg}$ ) was extracted with $70 \%$ ethanol by the reflux method twice; and each lasted $1 \mathrm{~h}$. The mixture was filtered to collect the filtrate, which was evaporated to almost dryness using a rotary evaporator under reduced pressure. The residue was finally lyophilised to dryness to obtain the EE (yield $11.42 \%)$. The EE was then suspended in hot water and partitioned between ethyl acetate and water to obtain the ethyl acetate-soluble fraction (EAF) and the water-soluble fraction (WF). The two fractions were made into dried powders by a process of evaporation and lyophilisation.

Oleanolic acid and ursolic acid are the two main active compounds in FLL; and oleanolic acid is a commonly used chemical marker for the authentication of FLL according to the Chinese Pharmacopoeia (Edition 2010). HPLC detection of the two compounds in the EE of FLL and its two fractions, EAF and WF are shown in the Appendix. A C18 HPLC column $(4.6 \times 250 \mathrm{~mm}, 5 \mu \mathrm{m})$ was used in the HPLC analysis. A mobile phase consisting of $\mathrm{MeOH}-0 \cdot 1 \%$ acetic acid $(87: 13)$ was run at a flow rate of $1 \mathrm{ml} / \mathrm{min}$, and the detection wavelength was set at UV $204 \mathrm{~nm}$. Oleanolic acid and ursolic acid were adequately resolved from other unknown compounds and could be clearly identified by the retention time in the EE of FLL and its EAF (see Appendix (a)). WF was further analysed by HPLC under another condition (see Appendix (b)). A mobile phase consisting of acetonitrile and $0 \cdot 1 \%$ trifluoroacetic acid was run at a flow rate of $0.8 \mathrm{ml} / \mathrm{min}$, and the detection wavelength was set at UV $230 \mathrm{~nm}$. The linear gradient elution was set as follows: from the beginning, acetonitrile increased from 8 to $40 \%$ in $40 \mathrm{~min}$, and then increased to $70 \%$ in the next $20 \mathrm{~min}$. The two most prominent peaks in WF were identified to be nuezhenide and salidroside (see Appendix (b)).

\section{Animal study design}

A total of thirty-two 4-month-old mature (average weight 238.5 (SE 2.5)g) Sprague-Dawley female rats (Experimental Animal Center of the Hong Kong Chinese University, Hong Kong, China) were used in the first animal study. Upon acclimatisation with a medium-Ca diet (MCD; TD Teklad $98005,0.6 \% \mathrm{Ca}, 0.65 \% \mathrm{P}$ ) for $5 \mathrm{~d}$, the mature rats were randomly divided into four groups: the control group $(\mathrm{C}$, treated with vehicle (distilled water)) and FLL EE, FLL EAF or FLL WF as the treatment groups and pair-fed with MCD. The dosages of EE, EAF and WF were given according to their actual extraction ratio, namely 700,126 and $574 \mathrm{mg} / \mathrm{kg}$ per d, respectively. Total treatment period was 12 weeks. In the second experiment, the effects of the active fraction on Ca balance and bone properties were studied. A total of sixty 4-month-old mature Sprague-Dawley female rats (220-250 g; Experimental Animal Center of the Hong Kong Chinese University, Hong Kong, China) were randomly divided into three groups with differing dietary Ca levels and treated with either FLL water fraction (WF, $574 \mathrm{mg} / \mathrm{kg}$ per d) or its vehicle (distilled water) for 12 weeks. All rats were fed a MCD (TD $98005,0.6 \% \mathrm{Ca}, 0.65 \% \mathrm{P}$ ) for $5 \mathrm{~d}$ before the initiation of the treatment regimen. Diets containing different levels of Ca were low-Ca diet (LCD, TD 05004, 0.1\% Ca, 0.65\% P), MCD and high-Ca diet (HCD, TD $05005,1 \cdot 2 \% \mathrm{Ca}, 0.65 \% \mathrm{P}$ ). 
All diets were purchased from Harlan Teklad (Madison, WI, USA). All rats had free access to distilled water, and were fed $15 \mathrm{~g} / \mathrm{d}$ per rat of the respective diet, the minimum average food intake of the rats during the acclimation period. The body weight of the animals was recorded weekly. The rats were housed in a room that provided alternating $12 \mathrm{~h}$ of light and $12 \mathrm{~h}$ of darkness with the room temperature at $23 \pm 1{ }^{\circ} \mathrm{C}$ and humidity $55 \pm 5 \%$. Husbandry of the animals was based on the National Institutes of Health Guide for Care and Use of Laboratory Animals ${ }^{(15)}$. The experimental protocol was approved by the Animal Ethics Committee of The Hong Kong Polytechnic University.

\section{Sample collection}

The animals, $2 \mathrm{~d}$ before being killed, were housed individually in metabolic cages for collection of urine and faeces. On the day of killing, blood was withdrawn from the abdominal aorta under light diethyl ether anaesthesia, and the serum was prepared. Rat tibias were collected, cleaned of all soft tissue, wrapped in saline-soaked towels and stored at $-20^{\circ} \mathrm{C}$ for further analysis.

\section{Biochemical analysis of serum, urine and faeces samples}

Ca concentration in both serum and urine samples was measured using standard colorimetric methods with commercial kits (Wako Pure Chemical Industries Limited, Osaka, Japan). Urinary creatinine (Cr) was determined using the Jaffe method by kits (Wako Pure Chemical Industries Limited). Urinary $\mathrm{Ca}$ excretion was expressed as ratio of urinary $\mathrm{Ca}$ to $\mathrm{Cr}$ level. The faeces was first dried (at $110^{\circ} \mathrm{C}$ for $12 \mathrm{~h}$ ), then incinerated (at $800^{\circ} \mathrm{C}$ for $12 \mathrm{~h}$ ) in a muffle furnace and weighed. Faecal ash (50 mg) was then dissolved in $2 \mathrm{ml}$ of $6 \mathrm{M}-\mathrm{HCl}$ and diluted appropriately with Milli-Q water for atomisation. The amount of faecal $\mathrm{Ca}$ excretion in $24 \mathrm{~h}$ was determined by atomic absorption spectrophotometry (AAnalyst 100 Spectrometer; PerkinElmer, Waltham, MA, USA). The Ca absorption rate was calculated from the formula: $\mathrm{Ca}$ absorption rate $(\%)=($ intake $\mathrm{Ca}-$ faecal $\mathrm{Ca}) /$ intake $\mathrm{Ca} \times 100$; the $\mathrm{Ca}$ net balance was calculated from: $\mathrm{Ca}$ net balance $(\mathrm{mg})=$ intake $\mathrm{Ca}-$ faecal $\mathrm{Ca}$ - urine $\mathrm{Ca}$.

\section{Detection of calciotropic hormones}

Serum levels of intact PTH (1-84) were detected using rat bioactive intact PTH ELISA assay (Immutopics, Inc., San Clemente, CA, USA). Serum $1,25(\mathrm{OH})_{2} \mathrm{D}_{3}$ was extracted with two separate extraction columns and measured by competitive enzyme immunoassay (Immundiagnostik AG, Bensheim, Germany).

\section{Micro-computed tomography analysis of rat tibia}

Left tibias were thawed at room temperature before testing. The bone properties of tibia were studied using cone-beam X-ray micro-computed tomography ( $\mu \mathrm{CT}$; vivaCT40; Scanco Medical AG, Basserdrof, Switzerland). The $\mu \mathrm{CT}$ images of the proximal metaphysis in the left tibia head were obtained by scanning at medium resolution with $21 \mu \mathrm{m}$ increments at $70 \mathrm{kVp}$ and $110 \mu \mathrm{A}$ with a tube voltage of $50 \mathrm{kV}$, tube content of $0.1 \mathrm{~mA}$, slice thickness of $13 \mu \mathrm{m}$ and pixel size of $13 \mu \mathrm{m}$. The scanning process was controlled by an OpenVMS (Intel Itanium) workstation. The scanning positions of the tibia head were $2 \cdot 5-7 \mathrm{~mm}$ from the knee joint. A total of 210 consecutive slices from metaphysis bone were obtained for evaluation. After evaluation, a $\mu \mathrm{CT}$ reconstruction model was generated and three dimensional bone parameters were calculated by software. Bone content parameters included apparent bone mineral density, bone mineral content and bone volume: tissue volume ratio (BV:TV). Bone structural parameters included trabecular number, trabecular thickness, trabecular separation, bone surface:bone volume ratio (BS:BV) and connectivity density.

\section{Statistical analysis}

Data from these experiments were reported as means with their standard errors. All statistical analyses were performed using PRISM version 4.0 (GraphPad Software Inc., La Jolla, CA, USA). Analysis of the effects of diet, herb and interaction of both factors as grouping variables was performed by two-way ANOVA. Inter-group differences were analysed by unpaired $t$-test as a post-test. Differences in $P$ value of less than $0 \cdot 05$ were considered statistically significant.

Table 1. Effects of the ethanol extract (EE) of Fructus Ligustri Lucidi (FLL) and its fractions on serum or urine chemistries in mature female rats fed a medium-calcium diet (MCD; $0.6 \%$ calcium, $0.65 \%$ phosphorus) for 12 weeks

(Mean values with their standard errors, $n 6-8$ )

\begin{tabular}{|c|c|c|c|c|c|c|c|c|c|c|c|c|c|c|}
\hline & \multicolumn{2}{|c|}{$\begin{array}{l}\text { Weight } \\
\text { gain }(\mathrm{g})\end{array}$} & \multicolumn{2}{|c|}{$\begin{array}{c}\text { Serum } \\
\mathrm{Ca}(\mathrm{mg} / \mathrm{l})\end{array}$} & \multicolumn{2}{|c|}{$\begin{array}{l}\text { Serum } \\
P(m g / l)\end{array}$} & \multicolumn{2}{|c|}{$\begin{array}{l}\text { Urinary Ca:Cr } \\
\text { (mg/mg) }\end{array}$} & \multicolumn{2}{|c|}{$\begin{array}{l}\text { Urinary } \mathrm{P}: \mathrm{Cr} \\
(\mathrm{mg} / \mathrm{mg})\end{array}$} & \multicolumn{2}{|c|}{$\begin{array}{c}\text { Serum } \\
1,25(\mathrm{OH})_{2} \mathrm{D}_{3} \\
(\mathrm{pg} / \mathrm{ml})\end{array}$} & \multicolumn{2}{|c|}{$\begin{array}{l}\text { Serum PTH } \\
\quad(\mathrm{pg} / \mathrm{ml})\end{array}$} \\
\hline & Mean & SEM & Mean & SEM & Mean & SEM & Mean & SEM & Mean & SEM & Mean & SEM & Mean & SEM \\
\hline$C$ & 31 & 5 & 101.9 & 4.3 & 52.9 & 1.8 & 0.10 & 0.11 & 2.07 & 0.13 & 36.49 & $2 \cdot 87$ & $96 \cdot 19$ & 14.77 \\
\hline EE & 27 & 6 & 102.4 & 1.6 & 50.3 & 1.9 & $0.06^{*}$ & 0.01 & $1 \cdot 32$ & 0.13 & $44 \cdot 19$ & 3.37 & 110.6 & 23.01 \\
\hline EAF & 27 & 5 & $99 \cdot 2$ & 1.9 & 53.0 & 1.5 & 0.11 & 0.01 & 1.68 & 0.19 & 33.35 & 3.78 & $90 \cdot 71$ & 14.99 \\
\hline WF & 26 & 4 & $100 \cdot 8$ & $3 \cdot 1$ & $47 \cdot 1$ & 1.5 & $0.06^{*}$ & 0.01 & 1.35 & 0.07 & $36 \cdot 25$ & 4.96 & $135 \cdot 71$ & 14.76 \\
\hline
\end{tabular}

$\mathrm{Cr}$, creatinine; $1,25(\mathrm{OH})_{2} \mathrm{D}_{3}, 1,25$-dihydroxyvitamin $\mathrm{D}_{3} ; \mathrm{PTH}$, parathyroid hormone; $\mathrm{C}$, vehicle-treated group; EAF, ethyl acetate-soluble fraction in the EE of FLL; WF, water fraction in the EE of FLL.

${ }^{*}$ Mean values were significantly different from those of the vehicle-treated group $(P<0.05)$. 


\section{Results}

Effects of Fructus Ligustri Lucidi fractions on body weight, serum and urine chemistries

Body weight in the mature female rats increased gradually within the experimental period and the FLL treatment did not alter the weight gain (Table 1). Serum $\mathrm{Ca}$ and $\mathrm{P}$ were not altered by FLL treatment in mature female rats (Table 1). Urinary Ca:Cr was significantly decreased in rats by treatment with FLL EE and FLL WF $(P<0.05 v$. C, Table 1). Urinary P:Cr levels were not significantly altered in rats upon treatment with $\mathrm{EE}$ and its fractions (Table 1). EE appeared to increase serum $1,25(\mathrm{OH})_{2} \mathrm{D}_{3}$ levels; however, the increase was not statistically significant (Table 1). Similarly, serum PTH levels tended to increase in rats upon EE and WF treatment, but the increase did not reach statistical significance (Table 1).

\section{Effects of Fructus Ligustri Lucidi fractions on calcium} balance

Faecal $\mathrm{Ca}$ excretion in rats was significantly decreased by EE and its WF treatment $(P<0.05 v$. C, Fig. 1(a)). Both the calculated $\mathrm{Ca}$ net balance and $\mathrm{Ca}$ absorption rate in the mature rats were enhancfed simultaneously by EE and WF treatment $(P<0.05$ and $P<0.01 v$. C, respectively; Fig. 1(b) and (c)).

\section{Effects of Fructus Ligustri Lucidi water fraction} on body weight, serum and urine calcium and phosphorus levels in rats fed with different levels of dietary calcium

Body weight increased slowly in all six rat groups during the experimental period and weight gain in mature rats was not significantly altered in response to treatment with FLL WF (Table 2). However, two-way ANOVA analysis indicated that dietary $\mathrm{Ca}$ level altered the weight gain significantly $(P<0.05$, Table 2$)$. In particular, LCD induced more weight gain in both vehicle- and WF-treated female rats (Table 2). Serum Ca did not alter in rats fed LCD or MCD in response to WF treatment (Table 2). However, HCD significantly increased serum $\mathrm{Ca}$ levels in the vehicle-treated rats $(P<0.05$ $v$. LCD-fed rats), while WF significantly suppressed the increase in serum Ca levels in the HCD-fed groups $(P<0.05$ $v$. vehicle-treated HCD-fed rats, Table 2). Furthermore, neither WF treatment nor Ca diets influenced serum $\mathrm{P}$ levels in our study (Table 2). WF treatment decreased urinary $\mathrm{Ca}$ :Cr in the rats that were fed diet containing higher levels of $\mathrm{Ca}$, especially in the HCD groups $(P<0.05 v$. vehicle-treated rats, Table 2). Urinary $\mathrm{Ca}$ :Cr was significantly increased with increasing dietary $\mathrm{Ca}$ levels as demonstrated by two-way ANOVA analysis $(P<0.001$, Table 2$)$. Similarly, urinary P:Cr in rats was shown to be significantly altered in response to dietary $\mathrm{Ca}(P<0.0001$, Table 2$)$. In addition, WF treatment further increased urinary $\mathrm{P}: \mathrm{Cr}$ in the LCD-fed rats $(P<0.05$ $v$. vehicle-treated rats, Table 2).
Effects of Fructus Ligustri Lucidi water fraction on calciotropic hormone levels in rats fed with different levels of dietary calcium

Two-way ANOVA analysis indicated that serum $1,25(\mathrm{OH})_{2} \mathrm{D}_{3}$ levels in rats were not altered by WF treatment (Table 2); however, they were significantly influenced by the levels of dietary Ca $\left(P<0 \cdot 0001\right.$, Table 2). As expected, serum $1,25(\mathrm{OH})_{2} \mathrm{D}_{3}$ levels increased in rats fed LCD compared with those fed
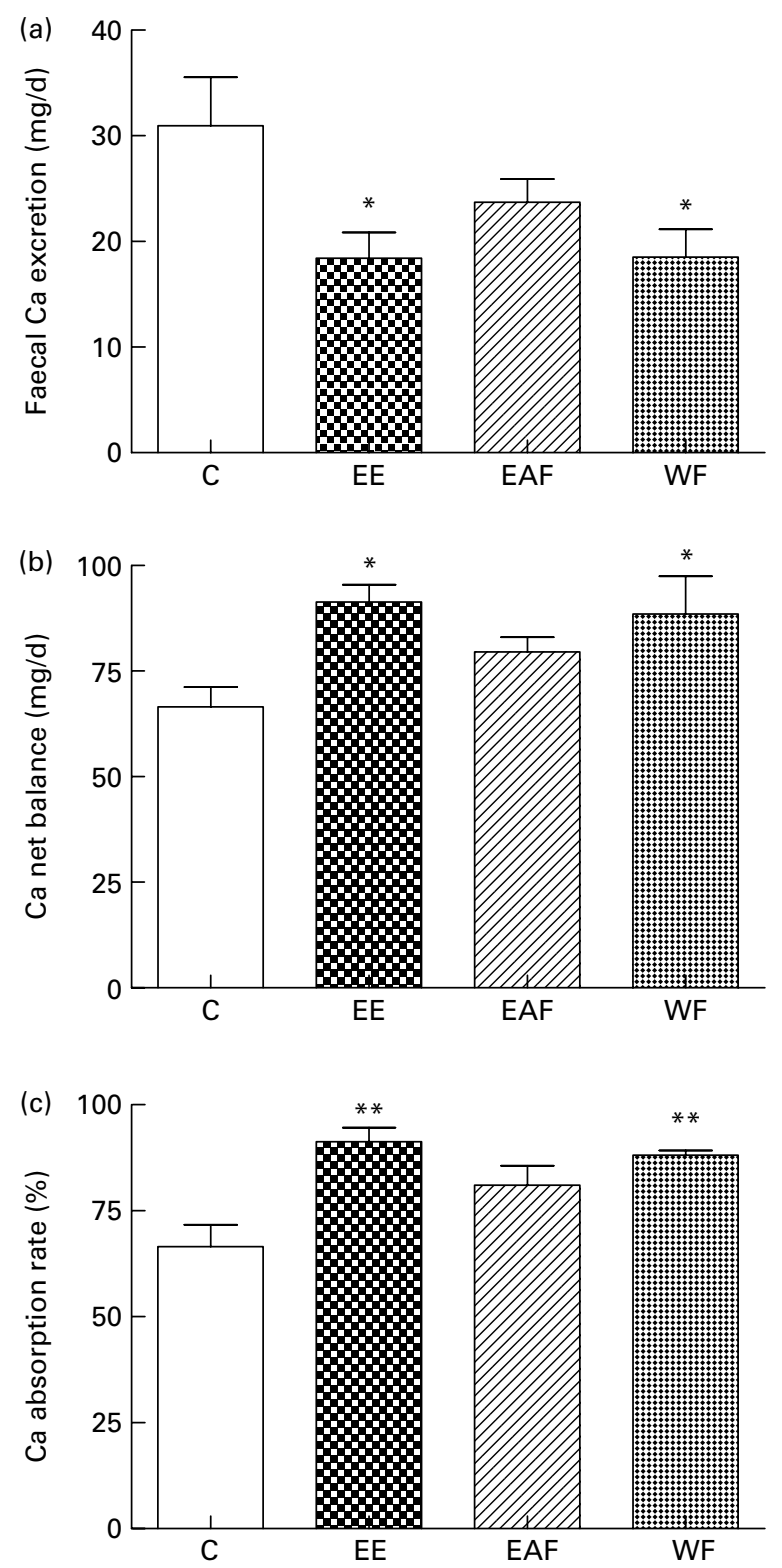

Fig. 1. Contents of (a) faecal $\mathrm{Ca}$, (b) Ca net balance and (c) Ca absorption rate in each treatment group. Sprague-Dawley female rats (4 months old) were treated with vehicle (C; distilled water) and the ethanol extract (EE), ethyl acetate-soluble fraction (EAF) or water fraction (WF) of Fructus Ligustri Lucid (FLL), at the dosage of 700,126 and $574 \mathrm{mg} / \mathrm{kg}$ per d, respectively. Total treatment period was 12 weeks. At the end of experiment, faeces and urine were collected for detection of $\mathrm{Ca}$ content. $\mathrm{Ca}$ net balance and $\mathrm{Ca}$ absorption rate were calculated according to their faecal and urinary $\mathrm{Ca}$ excretion. Values are means with their standard errors represented by vertical bars $(n 6-8)$. Mean values were significantly different from those of vehicle-treated group: ${ }^{*} P<0.05,{ }^{\star *} P<0.01$. 
MCD or HCD $(P<0.05, P<0 \cdot 01$, respectively; Table 2$)$. In contrast, WF treatment significantly increased serum PTH levels in MCD- and HCD-fed rats $(P<0.05 v$. vehicle-treated rats; Table 2). Furthermore, two-way ANOVA analysis indicated that the regulation of serum PTH was significantly altered by dietary $\mathrm{Ca}$, WF treatment as well as interaction between the dietary $\mathrm{Ca}$ and WF $(P<0 \cdot 05$, Table 2$)$.

Effects of Fructus Ligustri Lucidi water fraction on calcium balance in rats fed with different levels of dietary calcium

WF treatment appeared to suppress faecal Ca excretion in the MCD- and HCD-fed rats, though the suppression was not statistically significant (Fig. 2(a)). Net balance of Ca in vivo was significantly improved by WF treatment in both the MCDand HCD-fed rats $(P<0.05, P<0.01 v$. vehicle-treated rats, respectively; Fig. 2(b)). Similarly, the $\mathrm{Ca}$ absorption rate in the MCD- and HCD-fed rats was significantly elevated by WF treatment $(P<0.05$ and $P<0.01 \quad v$. vehicle-treated rats, respectively; Fig. 2(c)). In addition, high levels of dietary $\mathrm{Ca}$ significantly up-regulated faecal $\mathrm{Ca}$ and $\mathrm{Ca}$ net balance in both vehicle- and WF-treated mature female rats $(P<0.05$ and $P<0.001 v$. LCD-fed rats, respectively; Fig. 2(a) and (b)). Accordingly, the highest $\mathrm{Ca}$ absorption rate was found in the LCD-fed rats $(P<0.001 v$. MCD- and HCD-fed rats, Fig. 2(c)).

\section{Effects of Fructus Ligustri Lucidi water fraction on bone properties of rat tibia in rats fed with different levels of dietary calcium}

Dietary Ca significantly altered the parameters of bone content and structure in proximal metaphysis of the rat tibia. Our results showed that higher dietary Ca significantly increased trabecular number, trabecular thickness, connectivity density, apparent bone mineral density, bone mineral content and BV:TV $(P<0 \cdot 05$, Table 3$)$ and simultaneously decreased trabecular separation and BS:BV $(P<0.05$, Table 3$)$. Conversely, WF treatment seemed to induce negative alterations of bone parameters, including a slight decline of trabecular number, trabecular thickness, connectivity density, apparent bone mineral density, BV:TV and a slight increase of trabecular separation and BS:BV in all dietary groups; however, those slight effects on bone did not reach statistical significance. Two-way ANOVA analysis indicated that only dietary Ca level significantly influenced those bone parameters. Specifically, MCD and HCD were found to induce great improvement of apparent bone mineral density, bone mineral content and $\mathrm{BV} / \mathrm{TV}$ in mature female rats $(P<0.05$ and $P<0.01 v$. LCDfed rats, respectively; Table 3 ). WF treatment failed to induce apparent alteration of bone content in rats fed with different levels of dietary Ca (Table 3). Higher levels of dietary Ca could significantly ameliorate trabecular bone microarchitecture in rats through increasing trabecular numbers $(P<0.05 \quad v$. LCD-fed rats, Table 3$)$, thickness $(P<0.01$ $v$. LCD-fed rats, Table 3$)$ and decreasing separation $(P<0.05$ $v$. LCD-fed rats, Table 3) among them. Moreover, the ratio of bone surface area to total volume (BS:BV) decreased in rats 
in response to MCD or HCD ( $P<0.01 v$. LCD-fed rats, Table 3), suggesting a lower rate of bone resorption in these groups. Connectivity density in bone was improved by MCD and HCD treatment in rats $(P<0.05 v$. LCD-fed rats, Table 3$)$. Similarly, WF treatment did not result in any significant changes of bone microarchitecture in rats (Table 3 ).
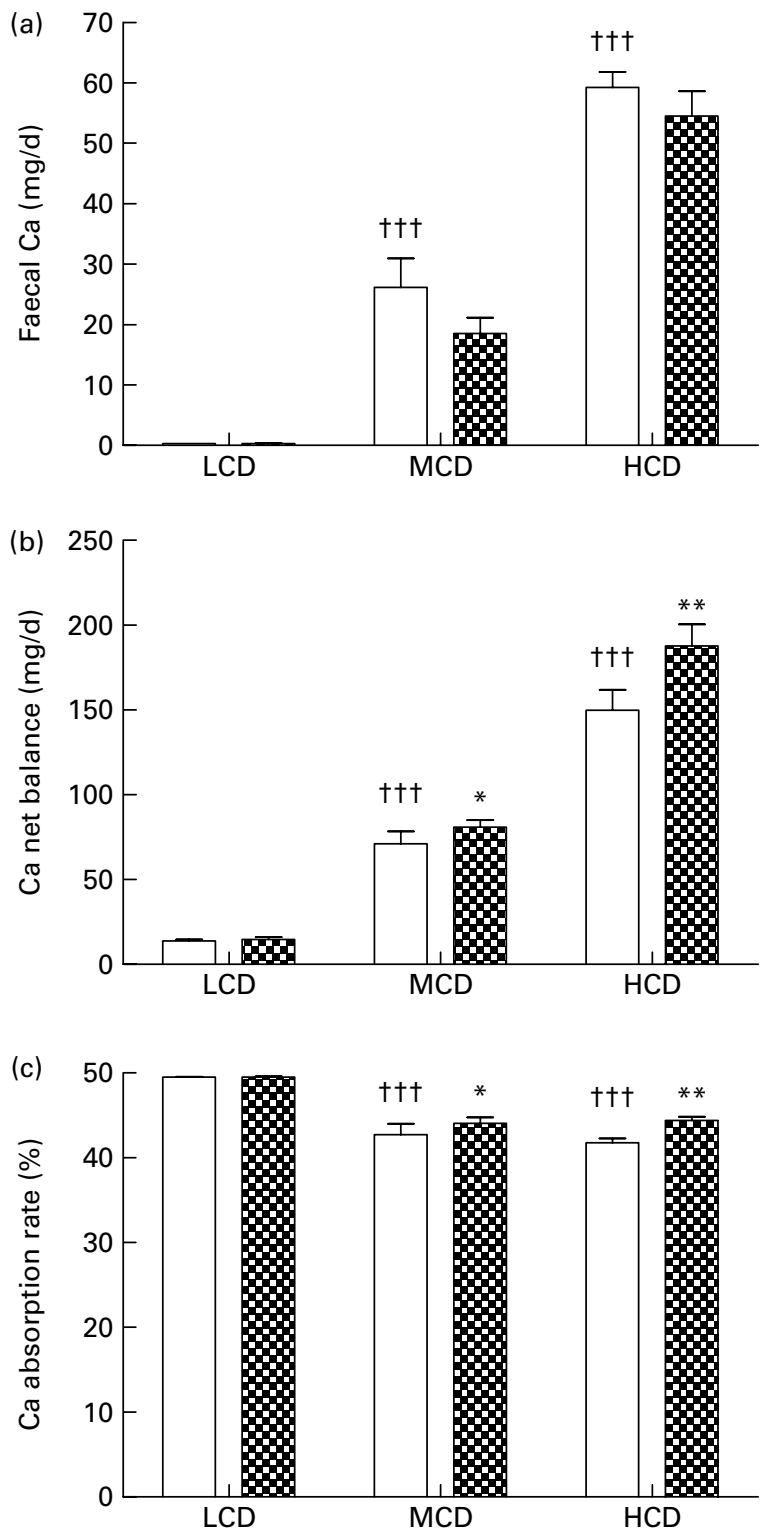

Fig. 2. Contents of (a) faecal $\mathrm{Ca}$, (b) Ca net balance and (c) Ca absorption rate in each group. Sprague-Dawley female rats (4 months old) were treated with vehicle ( $\square$; distilled water) and water fraction (WF, 囵) of Fructus Ligustri Lucidi at the dosage of $574 \mathrm{mg} / \mathrm{kg}$ per d, under three different levels of dietary $\mathrm{Ca}$ feeding. Total treatment period was 12 weeks. At the end of experiment, faeces and urine were collected for detection of $\mathrm{Ca}$ content. Ca net balance and $\mathrm{Ca}$ absorption rate were calculated according to their faecal and urinary $\mathrm{Ca}$ excretion. Values are means with their standard errors represented by vertical bars $(n 10)$. Mean values were significantly different from those of vehicle-treated group fed with a similar dietary $\mathrm{Ca}:{ }^{*} P<0.05$, ${ }^{* \star} P<0.01$. †††Mean values were significantly different from those of low-Ca diet (LCD; $0.1 \% \mathrm{Ca}, 0.65 \% \mathrm{P})$-fed rats in vehicle-treated group $(P<0.001)$. $\mathrm{MCD}$, medium-Ca diet $(0.6 \% \mathrm{Ca}, 0.65 \% \mathrm{P})$; $\mathrm{HCD}$, high-Ca diet $(1.2 \% \mathrm{Ca}$, $0.65 \% \mathrm{P})$.

\section{Discussion}

Our previous studies reported that FLL EE exerted positive effects on Ca metabolism in aged female rats ${ }^{(2)}$. The present study extended our previous findings to demonstrate that FLL (EE) could increase $\mathrm{Ca}$ balance and $\mathrm{Ca}$ absorption in mature female rats. Furthermore, WF from EE, but not EAF, exhibited a prominent effect on Ca balance, indicating that WF might be the active fraction of $\mathrm{EE}$ for Ca regulation. The subsequent experiment confirmed that WF could offer significant enhancing effects on Ca balance and absorption when the dietary Ca was adequate. The positive actions of WF on $\mathrm{Ca}$ balance were associated with the rise in serum PTH levels without altering serum $1,25(\mathrm{OH})_{2} \mathrm{D}_{3}$ levels in mature normal female rats. Furthermore, the increase in PTH levels by FLL treatment did not induce apparent bone loss in the mature-female-rat model. In addition, WF was found to help prevent surges of serum Ca levels with increasing dietary $\mathrm{Ca}$ in the mature female rats.

The mechanism of the actions of FLL on vitamin D metabolism in female rats appeared to alter with age. In contrast to our previous study ${ }^{(2)}$ in which serum $1,25(\mathrm{OH})_{2} \mathrm{D}_{3}$ levels increased in response to FLL treatment in aged female rats, serum $1,25(\mathrm{OH})_{2} \mathrm{D}_{3}$ levels did not alter in response to treatment with FLL EE and its fractions in the mature female rats. Previous studies by others ${ }^{(16,17)}$ showed that the levels of serum $1,25(\mathrm{OH})_{2} \mathrm{D}_{3}$ declined with age. Thus, it is possible that the lower background levels of serum $1,25(\mathrm{OH})_{2} \mathrm{D}_{3}$ in the aged rats allowed further stimulation of its biosynthesis by FLL in our previous study ${ }^{(2)}$. In contrast, the regulation of $1,25(\mathrm{OH})_{2} \mathrm{D}_{3}$ biosynthesis in mature rats might be tightly regulated by its stimuli and further stimulation might be inhibited by the physiological feedback mechanisms ${ }^{(18)}$, thereby preventing further increase of serum $1,25(\mathrm{OH})_{2} \mathrm{D}_{3}$ levels by FLL in mature rats in the present study.

Our results showed that WF treatment did not alter serum Ca levels in the rats fed LCD or MCD. Serum Ca levels were even down-regulated in the HCD-fed rats in response to WF treatment. Thus, the results indicated that WF treatment could prevent the surge of serum $\mathrm{Ca}$ in rats in response to HCD feeding. Moreover, WF treatment improved Ca net balance and $\mathrm{Ca}$ absorption rate in both the MCD- and HCDfed rats through suppressing urinary and faecal $\mathrm{Ca}$ excretion. Most importantly, urinary Ca excretion was decreased greatly in the WF-treated HCD-fed rats. Thus, it was intriguing to find that serum Ca levels in WF-treated HCD fed rats were suppressed despite the induction of intestinal $\mathrm{Ca}$ absorption rate as well as the suppression of urinary $\mathrm{Ca}$ excretion by WF treatment in these animals.

Our results also showed that WF treatment significantly increased serum PTH levels in the MCD- and HCD-fed rats. It is well known that the hormonal regulation of PTH is complex and involves circulating Ca levels and $1,25(\mathrm{OH})_{2} \mathrm{D}_{3}{ }^{(19)}$. Low levels of extracellular Ca stimulate PTH secretion within minutes; while elevated levels of $\mathrm{Ca}$ inhibit hormone release and favour the degradation within parathyroid cells ${ }^{(20)}$. Another major regulator of PTH secretion is $1,25(\mathrm{OH})_{2} \mathrm{D}_{3}$ in which PTH mRNA expression in the parathyroid gland is 


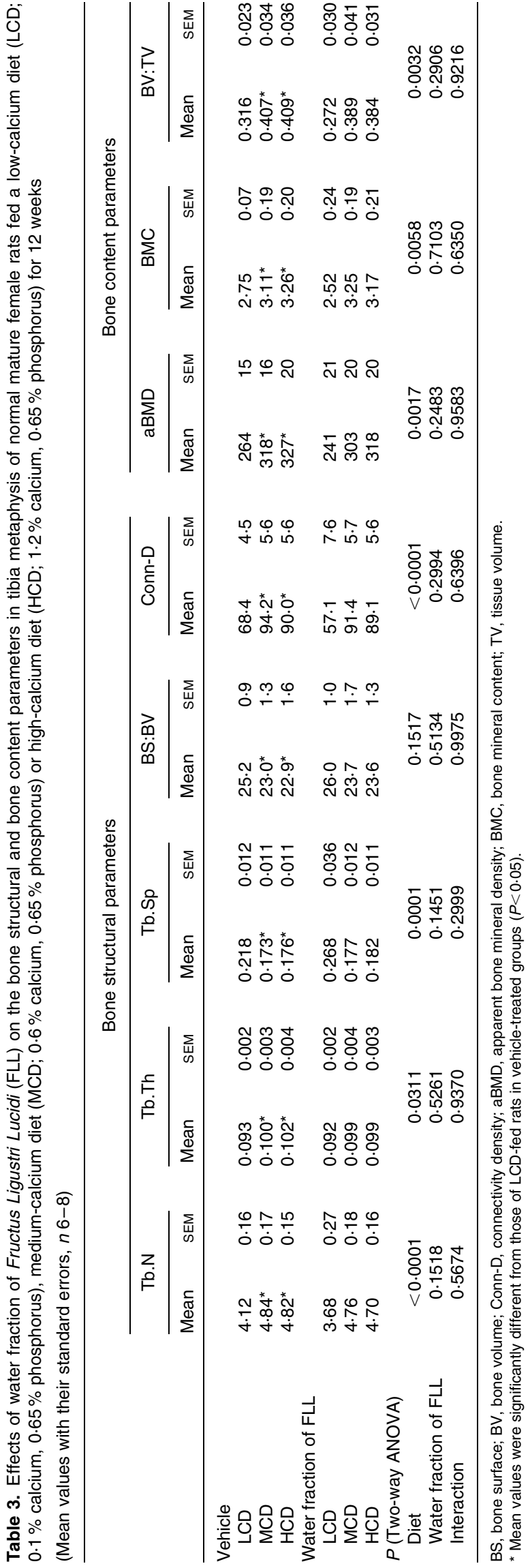

regulated by $1,25(\mathrm{OH})_{2} \mathrm{D}_{3}{ }^{(20)}$. In addition, $1,25(\mathrm{OH})_{2} \mathrm{D}_{3}$ is capable of indirectly inhibiting PTH secretion by increasing serum $\mathrm{Ca}$ through its actions on the intestine, bone and kidney ${ }^{(21)}$. As a result, serum PTH levels are normally induced in response to a prolonged decrease in serum $\mathrm{Ca}$ and $1,25(\mathrm{OH})_{2} \mathrm{D}_{3}$ levels, or an increase in serum $\mathrm{P}$ levels ${ }^{(20)}$. However, apart from the significant down-regulation of serum $\mathrm{Ca}$ levels by WF treatment in the HCD-fed rats, neither serum $1,25(\mathrm{OH})_{2} \mathrm{D}_{3}$ levels nor serum $\mathrm{P}$ levels were altered in these animals in response to WF treatment. Thus, the dramatic increase in serum PTH levels in both the MCD- and HCDfed rats in response to WF treatment could not be explained by the changes in circulating levels of $\mathrm{Ca}, \mathrm{P}$ or $1,25(\mathrm{OH})_{2} \mathrm{D}_{3}$. Our results suggest that the components in WF might exert direct effects on the regulation of PTH levels, possibly by altering the sensitivity of the parathyroid glands to serum $\mathrm{Ca}$ and $1,25(\mathrm{OH})_{2} \mathrm{D}_{3}$. Future studies will be needed to investigate the mechanism by which WF modulates serum PTH levels.

Elevation of serum PTH levels normally will enhance $1,25(\mathrm{OH})_{2} \mathrm{D}_{3}$ production, decrease urinary $\mathrm{Ca}$ excretion and increase bone resorption, thereby restoring serum Ca levels. Our results showed that the increase in serum PTH levels in mature female rats by WF treatment resulted in a significant reduction of urinary $\mathrm{Ca}$ excretion without any induction of serum $1,25(\mathrm{OH})_{2} \mathrm{D}_{3}$ levels or bone resorption. The loss of association between serum PTH and serum $1,25(\mathrm{OH})_{2} \mathrm{D}_{3}$ levels was also reported in our previous study that the significant increase in PTH levels in mature ovariectomised rats fed LCD did not result in any further increase in serum $1,25(\mathrm{OH})_{2} \mathrm{D}_{3}$ levels ${ }^{(22)}$. However, it is of interest to find that the positive actions of WF treatment on Ca balance did not result in any increase in bone mass and bone properties in mature female rats in contrast to our previous reported study on aged female rats ${ }^{(22)}$.

It is well known that secondary hyperparathyroidism is associated with increased bone turnover, decreased bone mineral density and increased fracture risks. On the other hand, intermittent administration of PTH induces bone formation ${ }^{(24)}$. Daily injection of PTH as either intact hormone PTH (1-84) or the active fragment PTH (1-34) is an efficacious bone anabolic treatment option for osteoporosis patients ${ }^{(1,25,26)}$. Such injections lead to rapid and transient spikes in hormone exposure levels, a profile that is a prerequisite to form bone effectively ${ }^{(27)}$. Hence, the newly discovered oral compounds that triggered rapid, transient spikes in endogenous PTH levels in rats or humans would also benefit bone formation $^{(27,28)}$. Thus, it is intriguing to find that the increase in serum PTH by WF treatment did not result in any negative influence on bone properties in the tibia in mature female rats fed MCD or HCD. However, it should be noted that the time-course responses of PTH levels to daily treatment of WT were not characterised in the present study. Thus, it is possible that the elevation of PTH levels in the WF-treated rats was transient and not continuous; further study is needed to characterise the PTH profile upon treatment with WT in these rats.

The lack of negative effects of high serum PTH levels on bone could be related to the overall Ca status/balance of the 
WT-treated animals as well as the protective effects of WT on bone. The present study clearly demonstrated that WT fraction could improve Ca balance in rats fed MCD or HCD, with a significant reduction of urinary $\mathrm{Ca}$ excretion as well as positive effects on intestinal $\mathrm{Ca}$ absorption. Our previous study showed that FLL extract could exert direct effects on the mineralisation process in osteoblast-like cells ${ }^{(2)}$. Thus, the positive effects of WT on bone and Ca balance might counteract the negative effects of high PTH levels in rats in the present study; resulting in no obvious changes in bone properties in the WT-treated animals fed MCD or HCD. Future studies are needed to investigate the mechanism by which WF treatment exerts protective effects on bone in the midst of increased PTH levels in mature female rats.

Currently, the most well-studied non-vitamin D dietary factors that increase $\mathrm{Ca}$ balance in humans or animals are non-digestible saccharides (NDS), including monosaccharides $^{(29-33)}$ and fructo-oligosaccharides ${ }^{(34-38)}$. Several mechanisms that contribute to the actions of NDS have been proposed: (1) NDS could be fermented by residing bacteria to produce byproducts, such as SCFA in the caecum and colon to result in a decrease in intestinal $\mathrm{pH}$ that eventually leads to an increase in Ca solubility ${ }^{(39)}$; (2) in vitro studies suggested that NDS indirectly open tight junctions in the epithelial cells by increasing intracellular $\mathrm{Ca}$ ion concentration, which in turn activates paracellular $\mathrm{Ca}$ transport and benefit Ca absorption ${ }^{(40)}$; (3) NDS could increase active Ca transport by the activation of calbindin $9 \mathrm{k}$ in large intestine only ${ }^{(41)}$. Our previous results indicated that FLL increased $1,25(\mathrm{OH})_{2} \mathrm{D}_{3}$-dependent CaBP expression in duodenum ${ }^{(2)}$ as well as renal 1-hydroxylase expression ${ }^{(4)}$ in aged female rats. In addition, the present study clearly demonstrated that FLL increased serum PTH levels in mature female rats. Thus, the mechanism of actions involved in increasing Ca balance by FLL and by NDS appears to be different.

Although FLL also contains quantitative constituents of polysaccharides ${ }^{(12-14)}$, the active constituents in FLL that are responsible for $\mathrm{Ca}$ regulation might not be NDS, as the reported mechanism of NDS is different from that of FLL. Moreover, it was found that oleanolic acid and ursolic $\operatorname{acid}^{(9-11)}$, the commonly reported active ingredients in FLL, are not contained in the identified active fraction of WF in the present study. Further study is required to identify the active components in FLL that exert the reported positive effects on $\mathrm{Ca}$ balance in vivo. Nuzhenide and salidroside, two major compounds that can be isolated from WF, might be candidates for explorations in future studies.

In summary, our present study demonstrated that FLL EE could improve $\mathrm{Ca}$ absorption and $\mathrm{Ca}$ balance, independent of the ages of the female rats. WF might be responsible for the positive effects of $\mathrm{EE}$ in regulating $\mathrm{Ca}$ absorption and $\mathrm{Ca}$ balance. The beneficial effects of WF in the mature female rats when dietary $\mathrm{Ca}$ was sufficient might be mediated by its direct or indirect action on PTH production. Most importantly, the increase in PTH levels induced by WF treatment did not result in any bone loss and the surges of serum $\mathrm{Ca}$ levels associated with high Ca feeding can also be prevented by WF treatment in mature female rats. These results suggested that WF is a potential agent that can be administrated orally to improve Ca balance and might be useful for the prevention of osteoporosis.

\section{Acknowledgements}

The authors thank the State Key Laboratory of Chinese Medicine and Molecular Pharmacology for providing the support in carrying out the present study. This work was supported by the Research Studentship and Niche Area Research Grant from the Research Committee of The Hong Kong Polytechnic University (I-BB8N) as well as the Shenzhen Basic Research Fund. X.-L. D was responsible for most of the experimental work and for drafting the manuscript. M. Z. and K.-K. W. helped in the herb extraction experiment and Micro CT detection work. C.-T. C. and M.-S. W. were responsible for the supervision and direction of the whole project as well as the final review of the manuscript. All authors declare that there are no conflicts of interest.

\section{References}

1. Neer RM, Arnaud CD, Zanchetta JR, et al. (2001) Effect of parathyroid hormone (1-34) on fractures and bone mineral density in postmenopausal women with osteoporosis. $N$ Engl J Med 344, 1434-1441.

2. Zhang Y, Lai WP, Leung PC, et al. (2008) Improvement of Ca balance by Fructus Ligustri Lucidi extract in aged female rats. Osteoporos Int 19, 235-242.

3. Zhang Y, Leung PC, Che CT, et al. (2008) Improvement of bone properties and enhancement of mineralization by ethanol extract of Fructus Ligustri Lucidi. Br J Nutr 99, 494-502.

4. Dong XL, Zhang Y, Favus MJ, et al. (2010) Ethanol extract of Fructus Ligustri Lucidi increases circulating 1,25-dihydroxyvitamin $\mathrm{D}_{3}$ by inducing renal 25-hydroxyvitamin D-1alpha hydroxylase activity. Menopause 17, 1174-1181.

5. Ward DT, Maldonado-Perez D, Hollins L, et al. (2005) Aminoglycosides induce acute cell signaling and chronic cell death in renal cells that express the calcium-sensing receptor. J Am Soc Nephrol 16, 1236-1244.

6. Hoenderop JG, Nilius B \& Bindels RJ (2005) Calcium absorption across epithelia. Physiol Rev 85, 373-422.

7. Tudpor K, Teerapornpuntakit J, Jantarajit W, et al. (2008) 1,25-dihydroxyvitamin $\mathrm{D}(3)$ rapidly stimulates the solvent drag-induced paracellular calcium transport in the duodenum of female rats. J Physiol Sci 58, 297-307.

8. Fujita H, Sugimoto K, Inatomi S, et al. (2008) Tight junction proteins claudin- 2 and -12 are critical for vitamin D-dependent $\mathrm{Ca}^{2+}$ absorption between enterocytes. Mol Biol Cell 19, 1912-1921.

9. Yim TK, Wu WK, Pak WF, et al. (2001) Hepatoprotective action of an oleanolic acid-enriched extract of Ligustrum lucidum fruits is mediated through an enhancement on hepatic glutathione regeneration capacity in mice. Phytother Res 15, 589-592.

10. Shi L, Cai Z, Wu G, et al. (1998) RP-HPLC determination of water-soluble active constituents and oleanolic acid in the fruits of Ligustrum lucidum Ait. collected from various areas. Zhongguo Zhong Yao Za Zhi 23, 77-79, 127.

11. Li G, Zhang XA, Zhang JF, et al. (2010) Ethanol extract of Fructus Ligustri Lucidi promotes osteogenesis of mesenchymal stem cells. Phytother Res 24, 571-576. 
12. Qiu RL \& Li L (2008) Study on extraction methods of polysaccharides in Fructus Ligustri Lucidi. Chin Traditional Patent Med 30, 612-614.

13. Zhang ZM, Ge B, Xu AX, et al. (2006) Antisenile effect of polysaccharides from Fructus ligustri lucidi. Chin J Pharmacol Toxicol 20, 108-111.

14. Ruan H \& Lu Z (1999) Studies on immunomodulatory function of polysaccharide of Fructus Ligustri Lucidi. Zhongguo Zhong Yao Za Zhi 24, 691-693, 704.

15. Council NR (2006) Guide for the Care and the Use of Laboratory Animals. Washington, DC: National Academy Press.

16. Lai WP, Chau TS, Cheung PY, et al. (2003) Adaptive responses of 25-hydroxyvitamin D3 1-alpha hydroxylase expression to dietary phosphate restriction in young and adult rats. Biochim Biophys Acta 1639, 34-42.

17. Armbrecht HJ, Boltz MA \& Bruns ME (2003) Effect of age and dietary calcium on intestinal calbindin D-9k expression in the rat. Arch Biochem Biophys 420, 194-200.

18. Dusso AS, Brown AJ \& Slatopolsky E (2005) Vitamin D. Am J Physiol Renal Physiol 289, F8-F28.

19. Carrillo-Lopez N, Fernandez-Martin JL \& Cannata-Andia JB (2009) The role of calcium, calcitriol and their receptors in parathyroid regulation. Nefrologia 29, 103-108.

20. Silver J, Kilav R \& Naveh-Many T (2002) Mechanisms of secondary hyperparathyroidism. Am J Physiol Renal Physiol 283, F367-F376.

21. Goodman WG (2004) The flavors of vitamin D: tasting the molecular mechanisms. Kidney Int 66, 1286-1287.

22. Zhang Y, Lai WP, Wu CF, et al. (2007) Ovariectomy worsens secondary hyperparathyroidism in mature rats during low-Ca diet. Am J Physiol Endocrinol Metab 292, E723-E731.

23. Zhang Y, Dong XL, Leung PC, et al. (2008) Fructus ligustri lucidi extract improves calcium balance and modulates the calciotropic hormone level and vitamin D-dependent gene expression in aged ovariectomized rats. Menopause 15, 558-565.

24. Qin L, Raggatt LJ \& Partridge NC (2004) Parathyroid hormone: a double-edged sword for bone metabolism. Trends Endocrinol Metab 15, 60-65.

25. Greenspan SL, Bone HG, Ettinger MP, et al. (2007) Effect of recombinant human parathyroid hormone (1-84) on vertebral fracture and bone mineral density in postmenopausal women with osteoporosis: a randomized trial. Ann Intern Med 146, 326-339.

26. Ettinger B, San Martin J, Crans G, et al. (2004) Differential effects of teriparatide on BMD after treatment with raloxifene or alendronate. J Bone Miner Res 19, 745-751.

27. John MR, Widler L, Gamse R, et al. (2011) ATF936, a novel oral calcilytic, increases bone mineral density in rats and transiently releases parathyroid hormone in humans. Bone 49, 233-241.
28. Kumar S, Matheny CJ, Hoffman SJ, et al. (2009) An orally active calcium-sensing receptor antagonist that transiently increases plasma concentrations of PTH and stimulates bone formation. Bone 46, 534-542.

29. Brommage R, Binacua C, Antille $S$, et al. (1993) Intestinal calcium absorption in rats is stimulated by dietary lactulose and other resistant sugars. J Nutr 123, 2186-2194.

30. Mitamura R, Hara H \& Aoyama Y (2004) Ingestion of raffinose promotes calcium absorption in the large intestine of rats. Biosci Biotechnol Biochem 68, 384-389.

31. Griessen M, Speich PV, Infante F, et al. (1989) Effect of absorbable and nonabsorbable sugars on intestinal calcium absorption in humans. Gastroenterology 96, 769-775.

32. Lacour B, Ohan J, Aznag A, et al. (1995) Stimulating effects of sorbitol and L-xylose on rat ileal Ca transport in vitro. Miner Electrolyte Metab 21, 391-397.

33. Tardivel S, Fournier P, Dupuis Y, et al. (1994) Stimulation of ileal calcium absorption by sorbitol, L-xylose, or creatine via a decrease in luminal sodium concentration: relation with concomitant changes in enterocyte energy metabolism. Calcif Tissue Int 54, 304-311.

34. Takahara S, Morohashi T, Sano T, et al. (2000) Fructooligosaccharide consumption enhances femoral bone volume and mineral concentrations in rats. $J$ Nutr $\mathbf{1 3 0}$, 1792-1795.

35. Morohashi T, Sano T, Ohta A, et al. (1998) True calcium absorption in the intestine is enhanced by fructooligosaccharide feeding in rats. J Nutr 128, 1815-1818.

36. Mineo H, Hara H, Kikuchi H, et al. (2001) Various indigestible saccharides enhance net calcium transport from the epithelium of the small and large intestine of rats in vitro. J Nutr 131, 3243-3246.

37. van den Heuvel EG, Schoterman MH \& Muijs T (2000) Transgalactooligosaccharides stimulate calcium absorption in postmenopausal women. J Nutr 130, 2938-2942.

38. Griffin IJ, Davila PM \& Abrams SA (2002) Nondigestible oligosaccharides and calcium absorption in girls with adequate calcium intakes. Br J Nutr 87, Suppl. 2, S187-S191.

39. Scholz-Ahrens KE, Schaafsma G, van den Heuvel EG, et al. (2001) Effects of prebiotics on mineral metabolism. Am J Clin Nutr 73, 459S-464S

40. Suzuki T \& Hara H (2004) Various nondigestible saccharides open a paracellular calcium transport pathway with the induction of intracellular calcium signaling in human intestinal Caco-2 cells. J Nutr 134, 1935-1941.

41. Takasaki M, Inaba H, Ohta A, et al. (2000) Dietary shortchain fructooligosaccharides increase calbindin-D9k levels only in the large intestine in rats independent of dietary calcium deficiency or serum 1,25 dihydroxy vitamin D levels. Int J Vitam Nutr Res 70, 206-213. 


\section{Appendix}

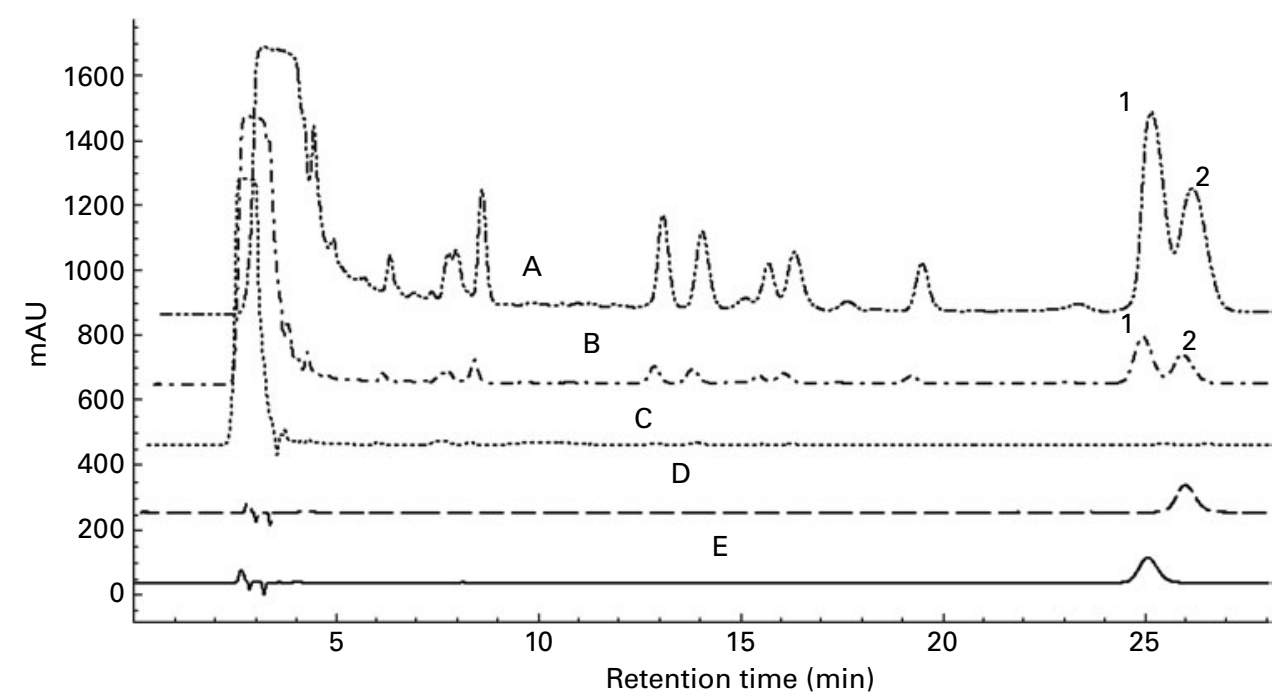

(a). Overlapped HPLC chromatograms for quantitative analysis of oleanolic acid and ursolic acid in the $70 \%$ ethanol extract of Fructus Ligustri Lucidi (FLL), and its sub-fractions. (A) Ethyl acetate fraction of FLL (EAF); (B) $70 \%$ ethanol extract of FLL (EE); (C) water fraction of FLL (WF); (D) ursolic acid standard; (E) oleanolic acid standard; (1) oleanolic acid; (2) ursolic acid.

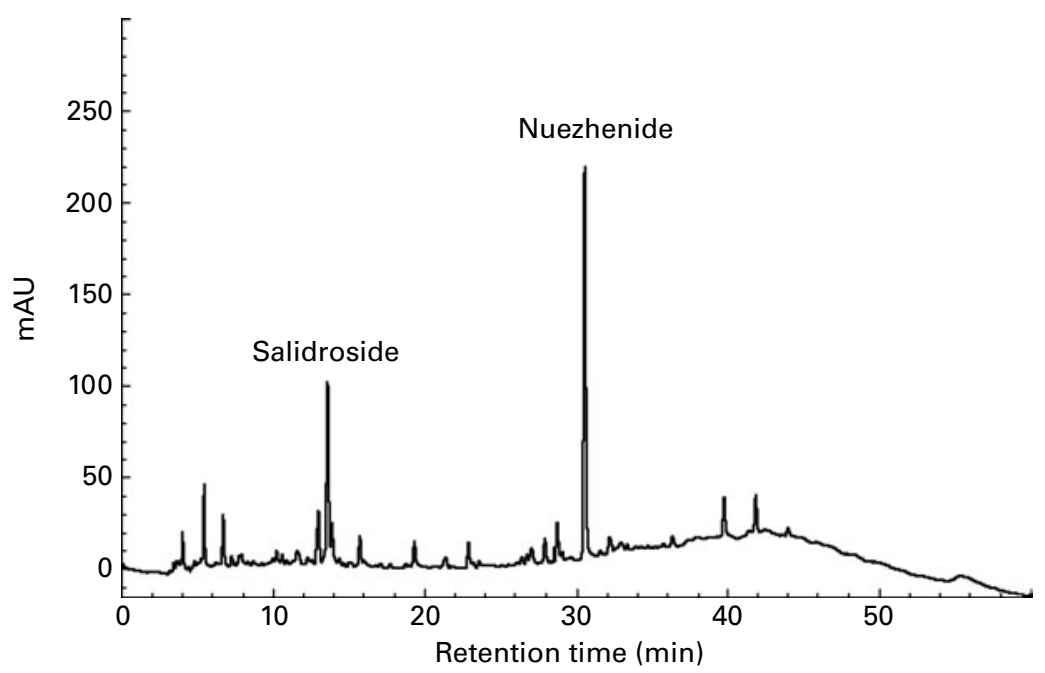

(b). HPLC profile of water-soluble fraction of Fructus Ligustri Lucidi run under a new condition. The two quantitative identified compounds in this fraction were salidroside and nuezhenide, which were shown in the figure, respectively. 